\title{
EVANESCENT OSCILLATIONS IN BUNCHING SYSTEMS
}

\author{
N.I. Ayzatsky, K.Yu. Kramarenko, S.A. Perezhogin \\ National Science Centre Kharkov Institute of Physics\&Technology
}

\begin{abstract}
At the initial stage of acceleration, it is often necessary to create an increasing distribution of accelerating electric field for improving the bunching process. Such distributions can be created by gradually decreasing the coupling coefficients between the resonant volumes. In such bunching structures charged particles are bunched and accelerated by the travelling wave with increasing amplitude. But there is another possibility of creating such increasing distributions. It is well known that in periodic structures there are two different in basis electromagnetic eigen-oscillations supported by metal walls without external currents and charges - propagating and evanescent. In forbidden bands (stopbands) electromagnetic oscillations transfer no energy in the direction of periodicity and have decreasing (increasing) dependence on the longitudinal coordinate. Results of our investigations of the properties of the electromagnetic oscillations in the stopbands of the periodic structures are represented. We discuss the usage of such oscillations in accelerating systems for the bunching and accelerating of electron beams.
\end{abstract}

\section{INTRODUCTION}

The output characteristics of the beam at the exit of the accelerator is considerably influenced by the parameters of the injector system. An injector system based on the standing wave gives posibility to form high quality electron bunches and to accelerate them up to enough high energies. In the standing-wave injector systems the klystron bunching mechanism can be combined with acceleration. For this purpose it is necessary to create a special field distribution in which amplitude increases from the initial injection point and there must be sufficiently long gaps without field. Such distribution can be achieved by a selection of coupling coefficients $[1,2]$. It was shown that there is the eigen oscillation in the periodic structure that corresponds for so nontrivial field distribution [3]. It is known that in the boundless periodic structure two eigen electromagnetic oscillations exist. In the passbands eigen oscillations represent travelling waves moving in the opposite direction. In the forbidden bands, which are located between the passbands, the eigen oscillations have another form. In these frequency intervals electromagnetic oscillations transfer no energy in the direction of periodicity and have decreasing or increasing character. In the bounded periodic structure it is possible to create the field distribution corresponding to the one (increasing or decreasing) eigen oscillation.

*psa@kipt.kharkov.ua
In the case of the smooth waveguide the increasing distribution can not be obtained under any circumstances. Under any boundary conditions in the smooth waveguide the amplitude of the increasing solution is always smaller than the amplitude of the decreasing one. This paper is focused on the properties of evanescent oscillations of two types in periodic structures. Results of the investigation of two bunching systems based on evanescent oscillations are described.

\section{CAVITY CHAIN}

Let's consider the chain of cylindrical cavities coupled through small irises in side walls. The distribution of amplitudes of $E_{010}$ oscillations in the cavity chain is described by the set of equations of the following form [4]:

$\left(\omega^{2}-\omega_{0}^{2}(1+2 \varepsilon)\right) A_{n}+\varepsilon \omega_{0}^{2} A_{n-1}+\varepsilon \omega_{0}^{2} A_{n+1}=0$

where $A_{n-1}, A_{n}, A_{n+1}$ - field amplitude in the $n-1, n$, $n+1$ cavities, $\varepsilon$ - coupling coefficient: $\varepsilon=\frac{2 a^{3}}{3 \pi J_{l}^{2}\left(\rho_{1}\right) b^{2} D}$, $D$ - length and $b$ - radius of the cavity, $\omega_{0}$ - resonant frequency. Equation (1) is true for the thin side walls. Equation (1) is a homogeneous difference equation of the second order with the constant coefficients. The general solution of linear homogeneous equation is equal to the sum of partial solutions with unknown constants:

$$
\begin{aligned}
& A_{n}=C_{1} \rho_{1}^{n}+C_{2} \rho_{2}^{n}, \\
& \text { where } \rho_{1,2}=\beta \pm \sqrt{\beta^{2}-1}, \beta=\frac{2 \varepsilon \omega_{0}^{2}+\omega_{0}^{2}-\omega^{2}}{2 \varepsilon \omega_{0}^{2}} .
\end{aligned}
$$

The forbidden zone is determined by the condition $|\beta|>1$. The chain under consideration has two forbidden zones - $\omega<\omega_{0}, \omega>\omega_{\pi}$. In the second forbidden zone $\left(\omega>\omega_{\pi}\right)$ the first partial solution $C_{1} \rho_{1}^{n} \quad\left(-1<\rho_{1}<0\right)$ corresponds to the decreasing amplitude distribution in the chain: $A_{n-1}>A_{n}>A_{n+1}$. In the case of $A_{n}=C_{2} \rho_{2}^{n}$ $\left(\rho_{2}<-1\right)$ the distribution of the field has the increasing character: $A_{n-1}<A_{n}<A_{n+1}$. In the bounded structure constants $C_{1}$ and $C_{2}$ are determined by the boundary conditions. If constant $C_{2}$ is equal to zero, then the decreasing distribution of the field is formed in the chain. The ratio $C_{2} / C_{1}$ can be obtained from equations for the first and last cavities:

$$
\frac{C_{2}}{C_{1}}=-\frac{\xi_{1} \rho_{2}^{2}-\rho_{2}\left(2 \xi_{1}-1\right)+\left(\xi_{1}-1\right)}{\xi_{1} \rho_{1}^{2}-\rho_{1}\left(2 \xi_{1}-1\right)+\left(\xi_{1}-1\right)} C_{1}=\eta_{1},
$$


$\frac{C_{2}}{C_{1}}=-\frac{\xi_{2} \rho_{1}^{2}-\rho_{1}\left(2 \xi_{2}-1\right)+\left(\xi_{2}-1\right)}{\xi_{2} \rho_{2}^{2}-\rho_{2}\left(2 \xi_{2}-1\right)+\left(\xi_{2}-1\right)} \frac{\rho_{1}^{N-1}}{\rho_{2}^{N-1}} C_{1}=\eta_{2} \frac{\rho_{1}^{N-1}}{\rho_{2}^{N-1}}$,

where $\xi_{1}=D_{1} / D, \xi_{2}=D_{N} / D, N-$ the number of cavities. The coefficient $\mathrm{C}_{2}$ equals zero if $\eta_{1}=0$ and $\eta_{2}=0$. To obtain this, the length of the boundary cavities must be equal:

$$
D_{1}=\frac{1}{1-\rho_{2}} D<\frac{D}{2}, D_{N}=\frac{1}{1-\rho_{1}} D>\frac{D}{2}
$$

To form the increasing field distribution $\left(C_{1}=0, \eta_{1} \rightarrow \infty, \eta_{2} \rightarrow \infty\right)$, the length of the first and last cavities must be selected in a such way:

$$
D_{1}=\frac{1}{1-\rho_{1}} D>\frac{D}{2}, D_{N}=\frac{1}{1-\rho_{2}} D<\frac{D}{2}
$$

Taking into account that $\rho_{1} \cdot \rho_{2}=1$ one can show that $D_{1}+D_{N}=D$.

\section{BIPERIODIC CAVITY CHAIN}

Consider the case when the cavity geometric sizes and the irises radii are altered periodically. We denote by $A_{n}$ the amplitudes of $E_{010}$ oscillation in the cavities with length $D_{1}$ and radius $b_{1}$, and by $B_{n}$ amplitudes in the cavities with length $D_{2}$ and radius $b_{2}$. We denote the iris radii by $a_{1}$ and $a_{2}$. In difference from the cavity chain considered above the amplitude distribution in the biperiodic cavity chain is described by two equations $[5,6]$ :

$$
\begin{aligned}
& \left(\omega_{1}^{2}\left(1+\alpha_{1}\right)-\omega^{2}\right) A_{n}=\omega_{1}^{2} \varepsilon_{1} B_{n}+\omega_{1}^{2} \varepsilon_{2} B_{n-1}, \\
& \left(\omega_{2}^{2}\left(1+\alpha_{2}\right)-\omega^{2}\right) B_{n}=\omega_{2}^{2} \varepsilon_{1} A_{n}+\omega_{2}^{2} \varepsilon_{2} A_{n+1},
\end{aligned}
$$

where

$$
\begin{aligned}
& \varepsilon_{1,2}=\frac{2}{3 \pi J_{1}^{2}\left(\lambda_{01}\right)} \frac{a_{1,2}^{3}}{b_{1} b_{2} \sqrt{D_{1} D_{2}}}, \\
& \alpha_{1,2}=\frac{2}{3 \pi J_{1}^{2}\left(\lambda_{01}\right)} \frac{a_{1}^{3}+a_{2}^{3}}{b_{1,2}^{2} D_{1,2}}, \omega_{1,2}=c \lambda_{01} / b_{1,2} .
\end{aligned}
$$

The amplitudes in $(\mathrm{n}+1)$ and $\mathrm{n}$ cavities are related by such conditions:

$$
A_{n+1}=\rho A_{n}, B_{n+1}=\rho B_{n}
$$

It is easy to show that at fixed frequency $\operatorname{Eqs}(7,8)$ have nonzero solutions for two values of the parameter $\rho$ :

$\rho_{1,2}=Q \pm \sqrt{Q^{2}-1}$,

where $\mathrm{Q}$ is determined by the expression:

$$
Q=\frac{\left(\omega_{1}^{2}\left(1+\alpha_{1}\right)-\omega^{2}\right)\left(\omega_{2}^{2}\left(1+\alpha_{2}\right)-\omega^{2}\right)}{2 \omega_{1}^{2} \omega_{2}^{2} \varepsilon_{1}^{2} \Delta}-\frac{1+\Delta^{2}}{2 \Delta} \text {. }
$$

The parameter $\Delta$ is equal to the ratio $a_{2}^{3} / a_{1}^{3}$. The analysis of the dependence of $Q$ on $\omega$ shows that the stopband appears inside the passband. The minimum value of $Q$ corresponds to the middle of the stopband:

$\omega_{*}^{2}=\frac{1}{2}\left[\omega_{1}^{2}\left(1+\alpha_{1}\right)+\omega_{2}^{2}\left(1+\alpha_{2}\right)\right]$

$Q\left(\omega_{*}\right)=\frac{\left(\omega_{1}^{2}\left(1+\alpha_{1}\right)-\omega_{2}^{2}\left(1+\alpha_{2}\right)\right)^{2}}{2 \omega_{1}^{2} \omega_{2}^{2} \varepsilon_{1}^{2} \Delta}-\frac{1+\Delta^{2}}{2 \Delta}$

If $a_{1}=a_{2}$ and the cavity frequencies (including the coupling shifts) tend to the mean frequency of the stopband:

$\omega_{1}^{2}\left(1+\alpha_{1}\right)=\omega_{2}^{2}\left(1+\alpha_{2}\right)=\omega_{*}^{2}$,

the intermediate forbidden zone vanishes and so-called structure compensation takes place $[5,6]$. In this case the creating of the increasing (decreasing) distribution becomes impossible. If $a_{1} \neq a_{2}$ the intermediate forbidden zone does not disappear under any circumstances. Condition (14) is reduced to the demand that the working frequency must be equal to the mean frequency of the forbidden zone. Under such consideration the ratio $A_{n} / B_{n}$ tends to infinity when $\rho \rightarrow \rho_{1}$. This is possible only in one case: $A_{n} \rightarrow 0$. When $\rho \rightarrow \rho_{2}$ the ratio $A_{n} / B_{n}$ tends to zero, i.e. $B_{n} \rightarrow 0$.

For both fundamental solutions the field distribution has the follows property: in a sequence of cavities located through one the amplitude of the field is equal to zero. For an increasing solution such cavities adjoin at the left to the diaphragm with large iris. As for the decreasing solution, zero field cavities adjoin at the left to the diaphragm with small iris.

If we want to create the condition for supporting the field distribution that corresponds to the decreasing fundamental solution, we must bound the biperiodic waveguide by cavities, which have at the left the diaphragm with large iris. One has to make some frequency shift in boundary cavities too. In this case, the amplitude of the increasing solution is zero. If we want to create the condition for supporting the field distribution that corresponds to the increasing fundamental solution, we must bound the biperiodic waveguide by cavities, which have at the left the diaphragm with small iris.

Thus, selecting in appropriate way boundary conditions for the bounded electrodynamic system, one can create the distribution of amplitudes that corresponds only to one fundamental solution. It has to be noted that it is possible only in the stopband. Within the passband such condition can not be fulfilled.

\section{SIMULATION}

Proceeding from the theory presented above, two injector systems based on evanescent oscillations were simulated using SUPERFISH [7] and PARMELA [8] codes. All simulations were held upon the electron beam initial energy $\mathrm{W}_{0}=25 \mathrm{keV}$ and current $50 \mathrm{~mA}$ with space charge forces taken into account. Peak value of on-axis electric field is $30 \mathrm{MV} / \mathrm{m}$. 
Waveguide section composed from five accelerating cells was taken for simulations of bunching system based on disk-loaded waveguide. It is well known that the diskloaded waveguide has many forbidden zones. As a working forbidden zone we have chosen the second forbidden zone of the symmetric wave. If we want to work in the forbidden zone, the conditions for the eigen frequency of the system to lay in the forbidden zone must be created. The simplest way of creating such situation is shifting the frequency of the last cell. In the second forbidden zone the phase shift per cell equals $\pi(\rho<0)$. The time-transit angle per cell for particle with energy $\mathrm{W}=\mathrm{W}_{0}$ was chosen equal to $\theta=\frac{\omega D}{v}=0.3 \pi$. As a result of simulations, the on-axis increasing field distribution was obtained. The results are shown in Fig. 1.

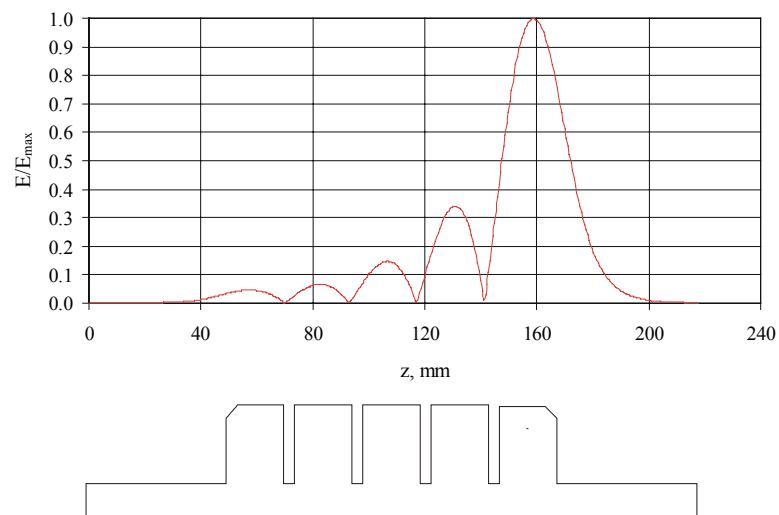

Figure 1: Geometry of bunching system base on diskloaded waveguide and corresponding on-axis electric field distribution

Electrodynamic performances of the simulated system are the follows: the quality factor $\mathrm{Q}=11163$, shunt impedance $\mathrm{R}_{\mathrm{sh}}=39.8 \mathrm{MOhm} / \mathrm{m}$. Simulation of particle dynamics in the system has shown that the maximum energy is $0.779 \mathrm{MeV}$, average energy is $0.697 \mathrm{MeV}$, energy spectrum is $9 \%$ ( $70 \%$ of particle), phase length is $32^{\circ}$, normalize emittance is $28 \mathrm{~mm} \cdot \mathrm{mrad}$ and capture is $91.2 \%$.

Waveguide section composed of eleven accelerating cells was taken for simulations of bunching system based on the biperiodic waveguide. The length of one cell is two times smaller than the length of the cell in the previous case. Biperiodic waveguide having all corresponding sizes to be equal except iris radii was considered. The time-transit angle for relativistic particle was chosen equal to $0.3 \pi$ per period. In this case the phase shift per period is $\pi$. The required on-axis increasing field distribution was obtained by periodical variations of iris radii and by boundary cell tunings. On-axis field distribution and geometry of a resonance system are shown in Fig 2.

Electrodynamic performances of the simulated system are the follows: the quality factor $\mathrm{Q}=5446$ and shunt impedance $R_{\mathrm{sh}}=10.8 \mathrm{MOhm} / \mathrm{m}$. Simulation of particle dynamics in the system has shown that the maximum energy is $0.7 \mathrm{MeV}$, average energy is $0.57 \mathrm{MeV}$, energy spectrum is $27 \%$ ( $70 \%$ of particle), phase length is $20^{\circ}$, normalize emittance is $18 \mathrm{~mm} \cdot \mathrm{mrad}$ and capture is $96 \%$.
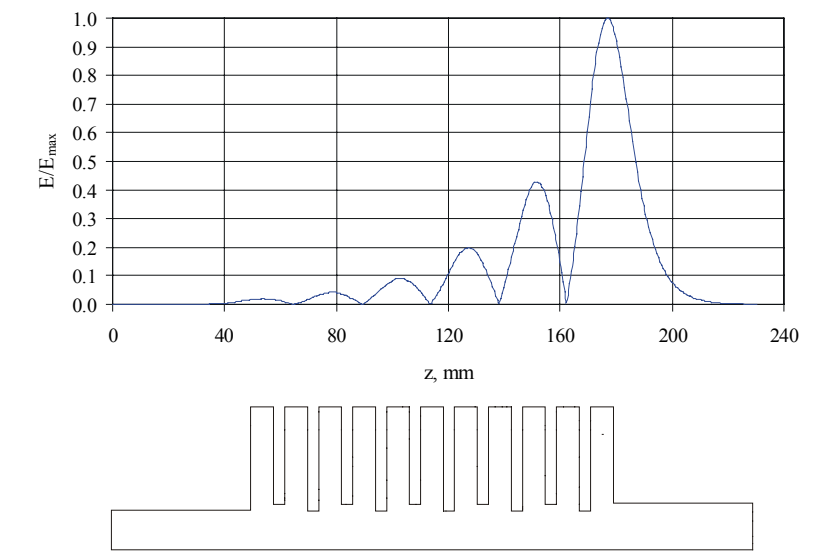

Figure 2: Geometry of bunching system base on ordinary biperiodical waveguide and corresponding on-axis electric field distripbution

\section{CONCLUSION}

Electron beam bunching process in periodic and biperiodic structures has been studied. The corresponding field distributions are based on the evanescent waves that exist in stopbands of the periodic structure. Two models of bunching systems were considered. The possibility of electron beam bunching and accelerating with good performances has been shown.

The authors would like to thank Kushnir V.A. and Mitrochenko V.V. for valuable discussions.

\section{REFERENCES}

[1] Zverev B.V., Timofeev V.F., Shilov V.K. Simulation and design of small-sized electron accelerator RELAC-3 Coll. Accelerator, M.: Energoatomizdat, 1981, p. 19-22.

[2] Vakhrushin Yu.P., Rjabtsov A.V., Smirnov V.L., Terentjev V.V. Acceleration structure with optimized many cavity buncher. Proceedings of the conference on charge particle accelerators, Dubna, 1993, 1, p. 249-251.

[3] Ayzatsky M.I. Electromagnetic oscillations in periodic mediums and waveguides outside the passband. Problems of atomic science and technoloy,1999, N3, p. 6-9.

[4] Bethe H.A. Theory of diffraction by small holes. Phys. Rev., 1944, 66, N7-8, p. 163-182.

[5] T. Nishikawa, S. Giordano, D. Carter. Dispersion relation and frequency characteristics of alternating periodic structure for linear accelerators. Rev. Sci. Instr. 1966, 37, N5, p. 652-661.

[6] D.E. Nagle, E.A. Knapp, B.C. Knapp. Coupled resonator model for standing wave accelerator tanks. Rev. Sci. Instr. 1967, 38, N11, p. 1583-1587.

[7] L.M. Young. "PARMELA", Los Alamos National Laboratory, LA-UR-96-1835, 1996.

[8] J.H. Billen and L.M. Young. POISSON/SUPERFISH on PC compatibles. Proc. 1993 Particle Accelerator Conff., Washington (USA), 1993, p. 790-792 